\title{
Determination of Heavy Metals in Locally Available Chocolates in Lahore Region
}

\author{
Muhammad Amjad ${ }^{1, a, *}$, Shabbir Hussain ${ }^{1, b}$, Zia Ur Rehman Baloch ${ }^{2, c}$, Aoun Raza ${ }^{1, d}$ \\ ${ }^{1}$ Department of Chemistry, Lahore Garrison University, Lahore-Pakistan \\ ${ }^{2}$ Department of Pharmacy University of Sargodha, Sargodha-Pakistan
}

*Corresponding author

A R T I C L I N F O A B S T R A T

Research Article

Heavy metal toxicity can either be acute or chronic effects. Long-term exposure of the body to heavy metal can progressively lead to muscular, physical and neurological degenerative process. In this research, a total of 30 representative chocolate samples were collected from local shops and markets in Lahore. All the samples were analysed to assess the levels of Lead (Pb), Nickel (Ni),

Received : 20/02/2021

Accepted : 14/03/2021 Chromium $(\mathrm{Cr})$ and Cadmium $(\mathrm{Cd})$ by using Atomic Absorption Spectrophotometer in Pakistan Council of Scientific and Industrial Research (PCSIR), Lahore. It was observed that the majority of chocolate samples contain heavy metals levels higher than the permissible limits as recommended by Punjab Food Rules (PFR) 2011. The levels of Pb ranged between 0.375 and $3.4 \mathrm{mg} / \mathrm{kg}$ with $90 \%$ samples having concentrations exceeding PFR allowable limit $(0.5 \mathrm{mg} / \mathrm{kg})$. Whereas, Ni concentrations varied from 0.005 to $0.28 \mathrm{mg} / \mathrm{kg}$ and $80 \%$ samples were above the PFR limit $(0.025$ $\mathrm{mg} / \mathrm{kg}$ ). Further, Cr levels analysed between 0.005 to $0.28 \mathrm{mg} / \mathrm{kg}$ and $53 \%$ samples had $\mathrm{Cr}$ concentrations higher than PFR limit $(0.02 \mathrm{mg} / \mathrm{kg})$. Similarly, Cd levels were observed between $0.50-3.25 \mathrm{mg} / \mathrm{kg}$ with $53 \%$ samples having Cd concentrations exceeding the PFR limit $(1.0 \mathrm{mg} / \mathrm{kg})$. Investigation indicates that the quality of available chocolates is not recommendable for eating

Keywords:

Heavy metals

Toxicity

Chocolate

Lahore

Atomic Absorption

\section{Introduction}

In the most recent decade, nourishment security has developed as a huge worldwide issue with general well being and pandemic exchange suggestions. Every person necessarily wants to have full of nutritious and secure foods at every place of living, so that they can make their families strong and a worth full individual of the community. Sustenance borne sicknesses related with microbial pathogens or other nourishment contaminants are a genuine wellbeing risk in creating and created nations (Sevindik et al., 2017; Mohammed et al., 2018). Flare-ups of such ailments can spread quickly and transnational excessively influence kids, the elderly. From all over the world billions of almost 1.5 situations of the run is happing every year, dominantly in the creations of nations, to have a sudden look upon almost 3 million of children aging 5 years. World Health Organisation (WHO) has assessed that $70 \%$ of dysentery scenes are caused by naturally defiled nourishment (Goldsteen et al., 2014; Mohammed et al., 2019).
Developing total populace and competiveness in exchange have offered ascend to the issue of nourishment wellbeing and security particularly in the poorer economies. Nourishment wellbeing issues without a doubt represent a major test to these countries, as they work to manage and enhance their monetary status. As per the WHO, sustenance borne sicknesses prevalently influence the economy of immature countries by adding to the weight of infection in the locale. The circumstance is elevated inferable from almost no observation and checking since a large portion of the instances of food borne sicknesses stay unreported and unrecognized. Thinking about this issue as a need, a greater part of the countries have started stern endeavours to control risks related with nourishment borne diseases particularly in the creating countries. Nourishment security issues in creating nations are generally perceived and are important to numerous worldwide associations. WHO has put nourishment security among its best 11 needs and is attempting huge endeavours to address the issue in the creating nations (Akhtar, 2015; Gizaw, 2019; Odeyemi et al., 2019). 
Newborn children and little youngsters in the creating nations are the most defenceless gatherings, with essential reasons of grimness and mortality in that gathering. Endeavours coordinated towards decreasing neediness and craving in the locales will clearly not work without a vital way to deal with enhance sustenance security circumstance. The main causes of the very much loose bowls and relevant difficulties, for example, kidney distress, incorporate Salmonella, Listeria, Campylobacter, or E. coli. Tuberculosis or brucellosis is transmitted through creatures and influence a generous section of the country populace associated with farming and domesticated animal administration (Kirk et al., 2015; Fong, 2017).

Sustenance borne sicknesses are viewed as a potential danger and key test as it mounts up the weight of illness in creating social orders. Evaluations demonstrate around 1500 diarrheal scenes happening universally, $75 \%$ of which are ascribed to organic defilement of nourishment and result in 3 million passings of youngsters matured 5 or less. In addition, organically tainted nourishments are considered as a reasonable determinant in the study of disease transmission of cholera and epidemic diarrhoea (Rivas et al., 2015). Aflatoxins, overwhelming metals, pesticide build-ups and various adulterants discover their way in the evolved way of life and harm the strength of countless in the creating scene, attributable to poor and inconsistent observing, reconnaissance, and control. These contaminants are answered to build the danger of growth and other interminable ailments in people. In like manner, inordinate exploitation of anti-infection agents to indulgence creatures has the outcome of expanding obstruction of microorganisms to these anti-toxins (Sevindik, 2019; Mohammed et al., 2020). Overwhelming metals display in the earth or following utilization of pesticides, are a noteworthy reason for disease and neurochemical harm (Lari et al., 2014).

Chocolate is a standout amongst the mainly ordinarily expended dessert worldwide prepared from cocoa as the standard crude material. The term 'cocoa' is a debasement of the expression 'cacao' this is taken instantly from Mayan and Aztec dialects. Chocolate is gotten from cocoa beans, applicable to the product of cocoa tree, Theobroma cacao that is native to South the unified states and established to have begun from the Amazon and Orinoco valleys (Alagić and Huremović, 2015).

The recorded scenery of chocolate begins in Mesoamerica. Developed beverages created utilizing chocolate backpedal to $350 \mathrm{BC}$. The Aztecs assumed that cacao seeds were the gift of Quetzalcoatl, the celestial power of intelligence, and the seeds once had so much regard that they were used as a sort of money. At first organized similarly as a drink, chocolate was filled in as an obnoxious, from a liquid, mixed with flavours or corn puree. It was acknowledged to have Spanish fly powers and to give the shopper quality. In recent days, these types of the relaxations are mostly termed as "Chilate and are to be come in existence by nearby humans persons in the South of Mexico. After its entrance to Europe in the sixteenth century, sugar was added to it, rendering it an affection mixture, and it ended up conspicuous all through society, first among the choice classes and subsequently among the normal subjects. In the twentieth century, chocolate was seen as fundamental in the extents of United States warriors at war (Verna, 2013).
Overwhelming metals are common components of world's structure; in any case purposeless human exercises have radically adjusted their geochemical cycles and biochemical change. This outcome in mixture of metals in plant parts having optional derivatives, which is in charge of a specific aetiological action. Pulled in out preface to captious metals, for example, cadmium, copper, lead, nickel, and zinc can results in malevolent flourishing effects in people. Substantial metals are among the most essential sorts of contaminant in the earth. Any destructive metal might be called critical metal, paying little personality to their nuclear mass or thickness. Noteworthy metals are a man from a not especially depicted cader of fragments that show alloy effects which hardens the altered metalloids, few of the substance has basic nature.

"Any metal (or metalloid) species might be viewed as a "contaminant" in the event that it happens where it is undesirable, or in a shape or focus that causes an adverse human or ecological impact. Metal/metalloid integrate Zinc $(\mathrm{Zn})$, Lead $(\mathrm{Pb})$, Strontium $(\mathrm{Sr})$ Cadmium $(\mathrm{Cd})$, Uranium (U), Mercury (Hg), Molybdenum (Mo), Arsenic (As), Manganese (Mn), Chromium (Cr), Cobalt (Co), Copper $(\mathrm{Cu})$, Cesium (Cs), Selenium (Se), Aluminium (Al), Nickel (Ni), and Silver (Ag)

In the current study, the main focus was to determine the existence of the selected heavy metals in the chocolates available in local markets of Lahore, manufactured in the local industries where there are no hygienic operating procedures are implementing. These types of contaminations not only found in only chocolates but also in other type of preferred eatables of kids e.g. candies, gums, juices etc. Heavy metal contamination is running the health of our kids badly causing sever health issues.

\section{Problem Statement}

High level of metals in food products like candies and chocolates are the cause of serious health problem worldwide. Candies and chocolates are the favourite food items for children all over the world including Pakistan; therefore, children are the vulnerable age group to get affected by heavy metal contamination. Excessive intake of heavy metals through these candies and chocolates is a great health concern as they accumulate over a lifetime in the body causing many health issues such as digestive problems, memory problems, headaches, anaemia, neurological disorders, slow growth, decrease in IQ, brain damage and many others. Therefore, in this project, concentration of selective heavy metals including Lead, Nickel, Cadmium and Chromium were examined in locally available chocolates of Lahore region.

\section{Delimitation of Study}

The presences of excessive levels of heavy metals also attributed to the manufacturing process of chocolates. For that reason, it was also significant to collect the data and related information about the in house conditions of the industries; how they are manufacturing the chocolate products. However, we could not get the permission from most of the factory owners to visit inside the manufacturing units. This could be because they were trying to hide their unhygienic manufacturing conditions. 


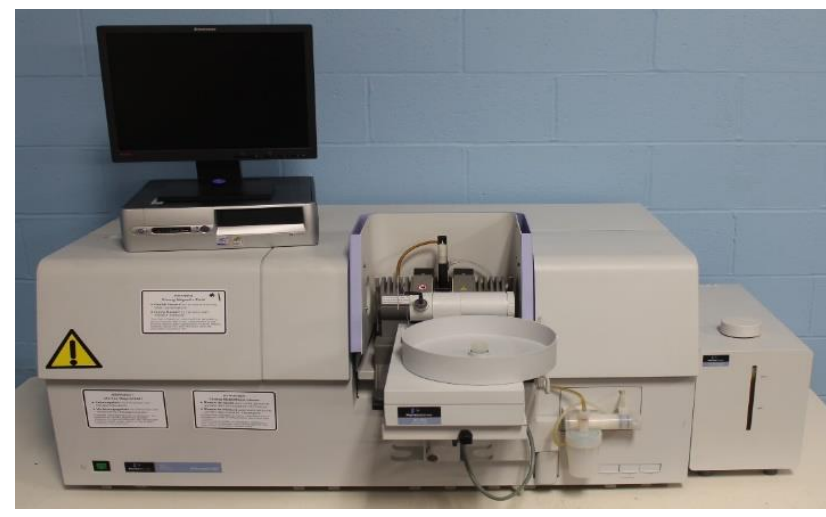

Figure 1. Atomic Absorption Spectrophotometer

\section{Limitation of Study}

- Time frame: minimum limit to complete this study was being 1 year; therefore, targets were set to complete the study within stipulated time.

- Financial constraints: due to constraint of resources and time, it was not possible to conduct the study on large scale.

- Man-power: the study was dealt single-handedly.

\section{Hypothesis}

Null Hypothesis

There is no significant content of Heavy Metals present in locally available chocolates.

Alternate Hypothesis

There is significant content of Heavy Metals present in locally available chocolates.

\section{Significance of Study}

The present study provided the information about contamination levels of toxic heavy metals, which could be helpful to understand the health risks associated with consumption of the locally manufactured chocolates.

\section{Objectives of Study}

- To determine the contents of heavy metals in locally available chocolate.

- To investigate the toxic effects of heavy metals in locally available chocolates in Lahore region.

\section{Materials and Methods}

\section{Location}

Present study was conducted to determine the heavy metals from locally available chocolates of Lahore region at Department of ACRC (Applied Chemistry Research Center), PCSIR Lahore.

\section{Procurement of Material}

All the chocolates were purchased from the local shops and departmental store of Lahore. All chemicals for heavy metals analysis were taken from the Applied Chemistry Research Center, PCSIR, and Lahore.

\section{Sampling}

Chocolates samples $(n=30)$ were collected from local shops and departmental stores of Lahore. Samples were analysed for heavy metals analysis at ACRC, PCSIR, Lahore.

\section{Instruments}

Samples of chocolate were analysed for heavy metals by Atomic Absorption spectrophotometer (Figure 1) supplied by Perkin Elmer, Model A Analyst 800, D2 background correction lamp. Acetylene-air flame was used for higher concentrations (mg/L).

\section{Preparation of Aqua Regia}

Mostly molar ratio between concentrated acids was $\mathrm{HCl}$ : $\mathrm{HNO}_{3}$ of 3:1. The used Hydrochloric acid was of $35 \%$, and Nitric acid was of $65 \%$, so 4 parts of $\mathrm{HCl}$ into 1 part of $\mathrm{HNO} 3$. 10 millilitre volumes is mostly used for all application. It is unusual to mix up a large volume of aqua regia.

Nitric Acid was added to the $\mathrm{HCl}$ by using hot plate to control temperature. Nitric Acid was poured to $\mathrm{HCl}$ instead of $\mathrm{HCl}$ to Nitric acid, a guming red or yellow liquid solution was resulted, which had given strong chlorine smell (Kanmani and Gandhimathi, 2013).

\section{Sample Preparation}

Firstly, the specific identification codes to all the chocolate samples were given in the symmetry as shown in Table 1.

\section{Sample Analysis}

Prior to start the analyses of heavy metals in the sample solutions, calibration of Atomic Absorption Spectrophotometer was done by checking $1^{\text {st }}$ blank sample then standard samples triplicates. The estimation of elemental concentration was determined by using Atomic Absorption Spectrophotometer using all the digested solutions. Finally, sample solutions were analysed on Atomic Absorption Spectrophotometer for heavy metals determination (Godbersen et al., 2012).

\section{Reagents}

All regents used in laboratory analysis were of analytical grades. Working standards of $\mathrm{Cd}, \mathrm{Ni}, \mathrm{Cr}$ and $\mathrm{Pb}$ were made by mixing to concentrated stock solutions (Merck, Darmstadt, Germany) of $1000 \mathrm{mg} / \mathrm{L}$ with 0.25 mol/L nitric acid (Chukwujindu MA Iwegbue, 2011).

\section{Results}

Locale chocolate samples $(n=30)$ were collected form retail shops, departmental stores of Lahore. Samples were analysed for heavy metals like lead, nickel, cadmium and chromium concentration. The determination of elemental concentration was determined by Atomic Absorption Spectrophotometer using the digested solutions (Godbersen et al., 2012). The analysed concentration levels of lead, nickel, cadmium and chromium are summarized in tables 2, 3, 4 and 5 respectively. The table 6 explains descriptive statistics of analytical results

\section{Lead $(\mathrm{Pb})$}

Lead $(\mathrm{Pb})$ was found to be the major heavy metal contaminant in chocolate samples with $90 \%$ samples exceeding Punjab Food Rules (PFR) 2011 permissible level $0.5 \mathrm{mg} / \mathrm{kg}$, and having range between 0.375 and $3.4 \mathrm{mg} / \mathrm{kg}$ with $1.4 \mathrm{mg} / \mathrm{kg}$ mean value (Table 2). 27 samples out of 30 had $\mathrm{Pb}$ concentration more than that of allowance level and only 1 chocolate sample had below the allowance level that is $0.375 \mathrm{mg} / \mathrm{kg}$, only 2 samples out of 30 samples had lead concentration equal to allowance value that is $0.5 \mathrm{mg} / \mathrm{kg}$. 
Table 1. The specific identification codes to all the chocolate samples

\begin{tabular}{|c|c|c|c|c|}
\hline Sr. & Brand Name & $\begin{array}{l}\text { Identification } \\
\text { Code }\end{array}$ & $\begin{array}{l}\text { Company Name, } \\
\text { Address }\end{array}$ & ents \\
\hline 1 & $\begin{array}{l}\text { Babble Milk } \\
\text { Chocolate }\end{array}$ & BMC-1 & $\begin{array}{l}\text { Funcity Food } \\
\text { Industries, Karachi }\end{array}$ & $\begin{array}{l}\text { Sugar, cocoa powder, milk powder, whey powder, vegetable fat, } \\
\text { soya lecithin, salt, vanillin }\end{array}$ \\
\hline 2 & $\begin{array}{l}\text { Excel Chico Liquid } \\
\text { Chocolate }\end{array}$ & ECLC-2 & $\begin{array}{l}\text { Naveed Enterprises } \\
\text { Faisalabad }\end{array}$ & $\begin{array}{l}\text { Sugar, hydrogenated vegetable fat, cocoa powder, skimmed milk, } \\
\text { emulsifier soya lecithin, GMS, vanilla powder, }\end{array}$ \\
\hline 3 & $\begin{array}{l}\text { Candyland Paradise } \\
\text { Coconut Filled } \\
\text { Chocolate }\end{array}$ & CPCFC-3 & $\begin{array}{l}\text { Ismail Industries } \\
\text { Limited, Hub }\end{array}$ & $\begin{array}{l}\text { th powder, hydrogenated vegetable } \\
\text { milk, powder, emulsifier soya }\end{array}$ \\
\hline 4 & $\begin{array}{l}\text { Spencer Milo Sip White } \\
\text { Liquid Chocolate }\end{array}$ & SMSWLC-4 & $\begin{array}{l}\text { D.F.I Pvt. Ltd., } \\
\text { Karachi }\end{array}$ & $\begin{array}{l}\text { Sugar, edible vegetable palm oil, milk solids, soya lecithin, } \\
\text { Artificial flavour, vanillin }\end{array}$ \\
\hline 5 & $\begin{array}{l}\text { GOFY Chocup Milk } \\
\text { chocolate Biscuit }\end{array}$ & GCMCB-5 & $\begin{array}{l}\text { KM Food Industries, } \\
\text { Lahore }\end{array}$ & $\begin{array}{l}\text { Sugar, hydrogenated vegetable fat, soya lecithin, ammonium bicarbonate, } \\
\text { salt, food colour, biscuits, wheat flour, cocoa powder, milk powder. }\end{array}$ \\
\hline 6 & $\begin{array}{l}\text { Giggly Squeezo } \\
\text { Chocolate Cream }\end{array}$ & GSCC-6 & Volka Food, Sahiwal & $\begin{array}{l}\text { Sugar, glucose, desiccatedcoconutpo } \\
\text { fat, sweet whey powder, skimmed m } \\
\text { lecithin, salt, artificial food flavour }\end{array}$ \\
\hline 7 & $\begin{array}{l}\text { Gofy Good Too Too } \\
\text { Milk Chocolate }\end{array}$ & GGTTMC-7 & $\begin{array}{l}\text { K.M Food Industries } \\
\text { Chohang, Lahore }\end{array}$ & $\begin{array}{l}\text { n bicarbonate, } \\
\text { powder. }\end{array}$ \\
\hline 8 & $\begin{array}{l}\text { City Boy Chocolate } \\
\text { Balls Chocolate Balls }\end{array}$ & СВСВСВ-8 & $\begin{array}{l}\text { Pearl Confectionary, } \\
\text { Karachi }\end{array}$ & whey powder, vegetable fat, \\
\hline 9 & Smart Cho Cone & SCC-9 & $\begin{array}{l}\text { Salman Food } \\
\text { Industries, Lahore }\end{array}$ & $\begin{array}{l}\text { dcoconutpowder, hydrogenated vegetable } \\
\text { skimmed milk, powder, emulsifier soya } \\
\text { d flavour }\end{array}$ \\
\hline 10 & $\begin{array}{l}\text { Bulbul Chocolate } \\
\text { Toffee }\end{array}$ & BCT-10 & Zubia Foods, Lahore & $\begin{array}{l}\text { le palm oil, milk solids, soya lecithin, } \\
\text { llin }\end{array}$ \\
\hline 11 & $\begin{array}{l}\text { Jhoom Jhoom Choco } \\
\text { Bars }\end{array}$ & JJCB-11 & $\begin{array}{l}\text { Nadir Borther Food } \\
\text { Industries, Lahore }\end{array}$ & $\begin{array}{l}\text { ilk powder, whey powder, vegetable fat, } \\
\text { lin }\end{array}$ \\
\hline 12 & Milko Choco Stick & MCS-12 & $\begin{array}{l}\text { Good Health } \\
\text { Foods,Lahore }\end{array}$ & $\begin{array}{l}\text { Sugar, glucose, desiccatedcoconutpowder, hydrogenated vegetable } \\
\text { fat, sweet whey powder, }\end{array}$ \\
\hline 13 & $\begin{array}{l}\text { Kiddy Chocolate } \\
\text { Spoon }\end{array}$ & KCS-13 & $\begin{array}{l}\text { Foodle Foods } \\
\text { Company, Lahore }\end{array}$ & $\begin{array}{l}\text { tedcoconutpowder, hydrogenated vegetable } \\
\text {, skimmed milk, powder, emulsifier soya } \\
\text { ood flavour }\end{array}$ \\
\hline 14 & Gamy Chocolate & GC-14 & $\begin{array}{l}\text { Nou } \\
\text { Foo }\end{array}$ & r, vegetable fat, \\
\hline 15 & Triangles Choc Bars & TCB-15 & Inam Foods, Lahore & k powder, whey powder, vegetable fat, \\
\hline 16 & Lahori Chocolate & LC-16 & $\begin{array}{l}\text { Waseem Company, } \\
\text { Lahore }\end{array}$ & $\begin{array}{l}\text { egetable } \\
\text { soya }\end{array}$ \\
\hline 17 & Cocoa Beans & CB-17 & ds, & $\begin{array}{l}\text { er, milk powder, whey pov } \\
\text { vanillin }\end{array}$ \\
\hline 18 & $\begin{array}{l}\text { Milky Chocolate } \\
\text { Khaoo }\end{array}$ & MCK-18 & $\begin{array}{l}\text { ndustries of } \\
\text { ahore }\end{array}$ & $\begin{array}{l}\text { lk powder, whey powder, vegetable fat, } \\
\text { in, artificial food flavour }\end{array}$ \\
\hline 19 & $\begin{array}{l}\text { Boom Boom Afridi } \\
\text { Chocolate }\end{array}$ & BBAC-19 & $\begin{array}{l}\text { Dhoom Dhoom } \\
\text { Industry, Lahore }\end{array}$ & $\begin{array}{l}\text { Sugar, cocoa powder, milk powder, whey powder, vegetable fat, } \\
\text { soya lecithin, salt, vanillin }\end{array}$ \\
\hline 20 & Best Choco Digit & BCD-20 & $\begin{array}{l}\text { Aslam Food Industry, } \\
\text { Lahore }\end{array}$ & $\begin{array}{l}\text { Sugar, cocoa powder, milk powder, whey powder, vegetable fat, } \\
\text { soya lecithin, salt, vanillin }\end{array}$ \\
\hline 21 & Cute Baby Chocolate & CBC-21 & $\begin{array}{l}\text { Meh } \\
\text { Com }\end{array}$ & $\begin{array}{l}\text { dcoconutpowder, hydrogenated vegetable } \\
\text { skimmed milk, powder, emulsifier soya } \\
\text { d flavour }\end{array}$ \\
\hline 22 & $\begin{array}{l}\text { Barbie Doll } \\
\text { Chocolates }\end{array}$ & BDC-22 & $\begin{array}{l}\text { Shahi Sweet Industry, } \\
\text { Lahore }\end{array}$ & $\begin{array}{l}\text { Sugar, cocoa powder, milk powder, whey powder, vegetable fat, } \\
\text { soya lecithin, salt, vanillin, artificial flavoured colour }\end{array}$ \\
\hline 23 & Kidco Chocolate & $\mathrm{KC}-23$ & $\begin{array}{l}\text { Nafees Foods Pvt., } \\
\text { Lahore }\end{array}$ & $\begin{array}{l}\text { Sugar, cocoa powder, milk powder, whey powder, vegetable fat, } \\
\text { soya lecithin, salt, vanillin }\end{array}$ \\
\hline 24 & $\begin{array}{l}\text { Need for Speed } \\
\text { Chocolate }\end{array}$ & NFSC-24 & ny of & regetable fat, \\
\hline 25 & $\begin{array}{l}\text { Micky Mouse Love } \\
\text { Chocolate }\end{array}$ & MMLC-25 & Sweets Product & $\begin{array}{l}\text { catedcoconutpowder, hydrogenated vegetable } \\
\text { ler, }\end{array}$ \\
\hline 26 & $\begin{array}{l}\text { Health and Wealth } \\
\text { Chocolate }\end{array}$ & HWC-26 & y Foods Pvt., & e fat, \\
\hline 27 & Doremon Chocolates & DC-27 & $\begin{array}{l}\text { Naeem industry Pvt. } \\
\text { Ltd., Lahore }\end{array}$ & $\begin{array}{l}\text { Sugar, cocoa powder, milk powder, whey powder, vegetable fat, } \\
\text { soya lecithin, salt, vanillin, artificial flavoured colour }\end{array}$ \\
\hline 28 & $\begin{array}{l}\text { Loving Mom } \\
\text { Chocolate }\end{array}$ & LMC-28 & $\begin{array}{l}\text { Farooq Sweets } \\
\text { Company, Lahore }\end{array}$ & $\begin{array}{l}\text { Sugar, glucose, desiccatedcoconutpowder, hydrogenated vegetable } \\
\text { fat, sweet whey powder, }\end{array}$ \\
\hline 29 & Delicious Chocolate & DC-29 & $\begin{array}{l}\text { Halal Foods Industry, } \\
\text { Lahore }\end{array}$ & $\begin{array}{l}\text { Sugar, cocoa powder, milk powder, whey powder, vegetable fat, } \\
\text { soya lecithin, salt, vanillin, artificial flavoured colour }\end{array}$ \\
\hline 30 & Silky Chocolate & SC-30 & $\begin{array}{l}\text { Rehmat Deserts } \\
\text { Company, Lahore }\end{array}$ & $\begin{array}{l}\text { Sugar, glucose, desiccatedcoconutpowder, hydrogenated vegetable } \\
\text { fat, sweet whey powder, }\end{array}$ \\
\hline
\end{tabular}


Table 2. Lead $(\mathrm{Pb})$ Concentrations in the Local brands of chocolates samples.

\begin{tabular}{|c|c|c|c|}
\hline$(\mathrm{n}=30)$ & Sample Codes Triplicate each sample & Mean Conc. mg/L & Concentration $\mathrm{ppm}^{*}$ \\
\hline 1 & BMC-1 & 0.0160 & 0.800 \\
\hline 2 & ECLC-2 & 0.0075 & 0.375 \\
\hline 3 & CPCFC-3 & 0.0150 & 0.750 \\
\hline 4 & SMSWLC-4 & 0.0680 & 3.400 \\
\hline 5 & GCMCB-5 & 0.0590 & 2.950 \\
\hline 6 & GSCC-6 & 0.0180 & 0.900 \\
\hline 7 & GGTTMC-7 & 0.0470 & 2.350 \\
\hline 8 & CBCBCB-8 & 0.0190 & 0.950 \\
\hline 9 & SCC-9 & 0.0120 & 0.600 \\
\hline 10 & BCT-10 & 0.0230 & 1.150 \\
\hline 11 & JJCB-11 & 0.0330 & 1.650 \\
\hline 12 & MCS-12 & 0.0200 & 1.000 \\
\hline 13 & KCS-13 & 0.0140 & 0.700 \\
\hline 14 & GC-14 & 0.0660 & 3.300 \\
\hline 15 & TCB-15 & 0.0560 & 2.800 \\
\hline 16 & LC-16 & 0.0180 & 0.900 \\
\hline 17 & CB-17 & 0.0110 & 0.550 \\
\hline 18 & MCK-18 & 0.0130 & 0.650 \\
\hline 19 & BBAC-19 & 0.0600 & 3.000 \\
\hline 20 & BCD-20 & 0.0270 & 1.350 \\
\hline 21 & CBC-21 & 0.0190 & 0.950 \\
\hline 22 & BDC-22 & 0.0380 & 1.900 \\
\hline 23 & $\mathrm{KC}-23$ & 0.0170 & 0.850 \\
\hline 24 & NFSC-24 & 0.0120 & 0.600 \\
\hline 25 & MMLC-25 & 0.0230 & 1.150 \\
\hline 26 & HWC-26 & 0.0100 & 0.500 \\
\hline 27 & DC-27 & 0.0100 & 0.500 \\
\hline 28 & LMC-28 & 0.0170 & 0.850 \\
\hline 29 & DC-29 & 0.0610 & 3.050 \\
\hline 30 & SC-30 & 0.0440 & 2.200 \\
\hline
\end{tabular}

PFR Maximum Allowance value is $0.5 \mathrm{mg} / \mathrm{kg}$ or ppm, ${ }^{*}$ Concentration in grams $\times 100 \mathrm{~mL}$

Table 3. Nickel (Ni) Concentration in the Local brands of chocolates samples.

\begin{tabular}{|c|c|c|c|}
\hline$(\mathrm{n}=30)$ & Sample Codes Triplicate each sample & Mean Conc. mg/L & Concentration $\mathrm{ppm} *$ \\
\hline 1 & BMC-1 & 0.00140 & 0.0700 \\
\hline 2 & ECLC-2 & 0.00170 & 0.0850 \\
\hline 3 & CPCFC-3 & 0.00210 & 0.1050 \\
\hline 4 & SMSWLC-4 & 0.00200 & 0.1000 \\
\hline 5 & GCMCB-5 & 0.00110 & 0.0550 \\
\hline 6 & GSCC-6 & 0.00090 & 0.0450 \\
\hline 7 & GGTTMC-7 & 0.00070 & 0.0350 \\
\hline 8 & CBCBCB-8 & 0.0050 & 0.0250 \\
\hline 9 & SCC-9 & 0.00460 & 0.2300 \\
\hline 10 & BCT -10 & 0.00110 & 0.0550 \\
\hline 11 & JJCB-11 & 0.00240 & 0.1200 \\
\hline 12 & MCS-12 & 0.00053 & 0.0265 \\
\hline 13 & KCS-13 & 0.00300 & 0.1500 \\
\hline 14 & GC-14 & 0.00190 & 0.0950 \\
\hline 15 & TCB-15 & 0.00200 & 0.1000 \\
\hline 16 & LC-16 & 0.03500 & 1.7500 \\
\hline 17 & CB-17 & 0.00500 & 0.2500 \\
\hline 18 & MCK-18 & 0.04100 & 2.0500 \\
\hline 19 & BBAC-19 & 0.003 & 0.15 \\
\hline 20 & BCD-20 & 0.0008 & 0.04 \\
\hline 21 & CBC-21 & 0.0005 & 0.025 \\
\hline 22 & BDC-22 & 0.00010 & 0.0050 \\
\hline 23 & $\mathrm{KC}-23$ & 0.00090 & 0.4500 \\
\hline 24 & NFSC-24 & 0.00080 & 0.0400 \\
\hline 25 & MMLC-25 & 0.00050 & 0.2500 \\
\hline 26 & HWC-26 & 0.00560 & 0.2800 \\
\hline 27 & DC-27 & 0.00020 & 0.0100 \\
\hline 28 & LMC-28 & 0.00010 & 0.0050 \\
\hline 29 & DC-29 & 0.00010 & 0.0050 \\
\hline 30 & SC-30 & 0.00080 & 0.0400 \\
\hline
\end{tabular}

PFR Maximum Allowance value is $0.025 \mathrm{mg} / \mathrm{kg}$ or $\mathrm{ppm},{ }^{*}$ Concentration in grams $\times 100 \mathrm{~mL}$ 
Table 4. Cadmium (Cd) Concentration in the Local brands of chocolates samples.

\begin{tabular}{|c|c|c|c|}
\hline$(n=30)$ & Sample Codes Triplicate each sample & Mean Conc.mg/L & Concentration $\mathrm{ppm}^{*}$ \\
\hline 1 & BMC-1 & 0.060 & 3.00 \\
\hline 2 & ECLC-2 & 0.060 & 3.00 \\
\hline 3 & CPCFC-3 & 0.059 & 2.95 \\
\hline 4 & SMSWLC-4 & 0.010 & 0.50 \\
\hline 5 & GCMCB-5 & 0.019 & 0.95 \\
\hline 6 & GSCC-6 & 0.065 & 3.25 \\
\hline 7 & GGTTMC-7 & 0.040 & 2.00 \\
\hline 8 & CBCBCB-8 & 0.020 & 1.00 \\
\hline 9 & SCC-9 & 0.015 & 0.75 \\
\hline 10 & BCT-10 & 0.024 & 1.2 \\
\hline 11 & JJCB-11 & 0.017 & 0.85 \\
\hline 12 & MCS-12 & 0.033 & 1.65 \\
\hline 13 & KCS-13 & 0.070 & 3.5 \\
\hline 14 & GC-14 & 0.023 & 1.15 \\
\hline 15 & TCB-15 & 0.020 & 1.00 \\
\hline 16 & LC-16 & 0.022 & 1.10 \\
\hline 17 & CB-17 & 0.019 & 0.95 \\
\hline 18 & MCK-18 & 0.011 & 0.55 \\
\hline 19 & BBAC-19 & 0.023 & 1.15 \\
\hline 20 & BCD-20 & 0.029 & 1.45 \\
\hline 21 & CBC-21 & 0.054 & 2.70 \\
\hline 22 & BDC-22 & 0.023 & 1.15 \\
\hline 23 & $\mathrm{KC}-23$ & 0.019 & 0.95 \\
\hline 24 & NFSC-24 & 0.023 & 1.15 \\
\hline 25 & MMLC-25 & 0.015 & 0.75 \\
\hline 26 & HWC-26 & 0.013 & 0.65 \\
\hline 27 & DC- 27 & 0.020 & 1.00 \\
\hline 28 & LMC-28 & 0.025 & 1.25 \\
\hline 29 & DC-29 & 0.013 & 0.65 \\
\hline 30 & SC-30 & 0.019 & 0.95 \\
\hline
\end{tabular}

Maximum Allowance value is $1.0 \mathrm{mg} / \mathrm{kg}$ or ppm, * Concentration in grams $\times 100 \mathrm{~mL}$

Table 5. Chromium (Cr) Concentration in the Local brands of chocolates samples.

\begin{tabular}{|c|c|c|c|}
\hline$(n=30)$ & Sample Codes Triplicate each sample & Mean Conc. mg/L & Concentration $\mathrm{ppm}$ \\
\hline 1 & BMC-1 & 0.00100 & 0.0500 \\
\hline 2 & ECLC-2 & 0.00090 & 0.0450 \\
\hline 3 & CPCFC-3 & 0.00170 & 0.0850 \\
\hline 4 & SMSWLC-4 & 0.00050 & 0.0250 \\
\hline 5 & GCMCB-5 & 0.00080 & 0.0400 \\
\hline 6 & GSCC-6 & 0.00210 & 0.1050 \\
\hline 7 & GGTTMC-7 & 0.00077 & 0.0380 \\
\hline 8 & CBCBCB-8 & 0.00560 & 0.2800 \\
\hline 9 & SCC-9 & 0.00040 & 0.0200 \\
\hline 10 & BCT-10 & 0.00010 & 0.0050 \\
\hline 11 & JJCB-11 & 0.00010 & 0.0050 \\
\hline 12 & MCS-12 & 0.00030 & 0.0150 \\
\hline 13 & KCS-13 & 0.00100 & 0.0500 \\
\hline 14 & GC-14 & 0.00020 & 0.0100 \\
\hline 15 & TCB-15 & 0.00010 & 0.0050 \\
\hline 16 & LC-16 & 0.00010 & 0.0050 \\
\hline 17 & CB-17 & 0.00070 & 0.0350 \\
\hline 18 & MCK-18 & 0.00067 & 0.0330 \\
\hline 19 & BBAC-19 & 0.00058 & 0.0290 \\
\hline 20 & BCD-20 & 0.00010 & 0.0050 \\
\hline 21 & CBC-21 & 0.00053 & 0.0265 \\
\hline 22 & BDC-22 & 0.00030 & 0.0150 \\
\hline 23 & $\mathrm{KC}-23$ & 0.00012 & 0.0100 \\
\hline 24 & NFSC-24 & 0.00010 & 0.0050 \\
\hline 25 & MMLC-25 & 0.00086 & 0.0430 \\
\hline 26 & HWC-26 & 0.00044 & 0.0220 \\
\hline 27 & DC-27 & 0.00070 & 0.0350 \\
\hline 28 & LMC-28 & 0.00020 & 0.0100 \\
\hline 29 & DC-29 & 0.00020 & 0.010 \\
\hline 30 & SC-30 & 0.00040 & 0.0200 \\
\hline
\end{tabular}

The maximum allowance value is $0.02 \mathrm{mg} / \mathrm{kg},{ }^{*}$ Concentration in grams $\times 100 \mathrm{~mL}$ 


\section{Nickel (Ni)}

The concentration of nickel (Ni) in locally available chocolates in Lahore region was also found to be very high. For instance, $80 \%$ samples were having the $\mathrm{Ni}$ concentrations higher than PFR acceptable level 0.025 $\mathrm{mg} / \mathrm{kg}$ and the range of concentrations was 0.005 to 2.05 $\mathrm{mg} / \mathrm{kg}$. As shown in Table 3, 24 samples out of 30 had Ni concentration more than that of allowance level, 4 sample chocolate had below the allowance level and 2 samples had nickel concentration equal to allowable limit.

\section{Cadmium $(C d)$}

The concentrations of cadmium (Cd) in locally available chocolates in Lahore region were also exceeding the PFR acceptable value $1.0 \mathrm{mg} / \mathrm{kg}$. 53\% samples were having the $\mathrm{Cd}$ concentrations higher than $1.0 \mathrm{mg} / \mathrm{kg}$ with values ranging between 0.5 and $3.5 \mathrm{mg} / \mathrm{kg}$. As Table 4 explains, 16 samples out of 30 had $\mathrm{Cd}$ concentration more than that of allowance level, only 11 sample chocolate had below the allowance level and 3 samples had $\mathrm{Cd}$ concentration equal to allowed limit $1.0 \mathrm{mg} / \mathrm{kg}$.

\section{Chromium (Cr)}

The concentration of chromium (Cr) in locally available chocolates in Lahore region found higher than the
PFR acceptable value $0.02 \mathrm{mg} / \mathrm{kg}$ in $53 \%$ samples. Table 5 shows $\mathrm{Cr}$ concentrations varied between 0.01 and 0.28 $\mathrm{mg} / \mathrm{kg}$. 16 samples out of 30 had $\mathrm{Cr}$ concentrations more than that of allowable limit and only 12 chocolate samples had concentrations below then the allowable level and 2 samples out of 30 samples had chromium concentration equal to allowance value that is $0.02 \mathrm{mg} / \mathrm{kg}$.

\section{Statistical Analysis}

Statistical analyses were done by using SPSS version 20 for the concentrations of Lead, Nickel, Chromium and Cadmium in all chocolate samples. Table 6 explains the descriptive statistics of analytical dataset. The mean concentrations of $\mathrm{Pb}, \mathrm{Ni}, \mathrm{Cd}$ and $\mathrm{Cr}$ are $1.423 \mathrm{mg} / \mathrm{kg}, 0.222$ $\mathrm{mg} / \mathrm{kg}, 1.438 \mathrm{mg} / \mathrm{kg}$ and $0.036 \mathrm{mg} / \mathrm{kg}$ respectively. It was noted that all mean concentrations of $\mathrm{Pb}, \mathrm{Ni}, \mathrm{Cd}$ and $\mathrm{Cr}$ are greater than PFR permissible limits $0.5 \mathrm{mg} / \mathrm{kg}, 0.025$ $\mathrm{mg} / \mathrm{kg}, 1.0 \mathrm{mg} / \mathrm{kg}$ and $0.02 \mathrm{mg} / \mathrm{kg}$ respectively. Figure 2 depicts the graphical representation of distribution of the analytical data. One-way ANOVA with Fisher's test (95\% confidence level) was carried out, which explains that there is a significance difference $(\mathrm{P}<0.05)$ between the mean values of $\mathrm{Pb}, \mathrm{Ni}, \mathrm{Cd}$ and $\mathrm{Cr}$ in chocolate samples (Table 7).

Table 6. Descriptive Statistics

\begin{tabular}{l|cccccc}
\hline & No. & Min. & Max. & Mean & Std. Deviation & Variance \\
\hline Lead $(\mathrm{Pb})$ & 30 & 0.38 & 3.40 & 1.423 & 0.972 & 0.945 \\
Nickel Ni) & 30 & 0.01 & 2.05 & 0.222 & 0.469 & 0.220 \\
Cadmium $(\mathrm{Cd})$ & 30 & 0.50 & 3.50 & 1.438 & 0.889 & 0.790 \\
Chromium $(\mathrm{Cr})$ & 30 & 0.01 & 0.28 & 0.036 & 0.052 & 0.003 \\
\hline
\end{tabular}

Table 7. Anova

\begin{tabular}{|c|c|c|c|c|c|}
\hline & Sum of Squares (SS) & Degrees of freedom (df) & Mean Square (MS) & $\bar{F}$ & $\mathrm{P}$ value \\
\hline Between Groups & 51.35 & 3 & 17.115 & 34.97 & 0.0 \\
\hline Within Groups & 56.78 & 116 & 0.489 & & \\
\hline Total & 108.13 & 119 & & & \\
\hline
\end{tabular}

\section{Discussion}

Sustenance security issues unquestionably a major test for creating nations. Sustenance wellbeing is major among top 11 needs of world wellbeing association, which is trying huge endeavours to illuminate the issue in the creating nations. Babies and little youngsters in the creating nations are the most powerless gatherings, with a central root of horribleness and mortality in kids. Sustenance borne sickness is seen as a potential danger and primary test in degenerating nations. Aflatoxins overwhelming metals, pesticide deposits and numerous adulterants are incorporated into the natural pecking order and damage the strength of monstrous number of individuals in the creating scene. These adulterants are accounted for to build the danger of malignancy and other perpetual sicknesses in humankind (Abnet, 2007; Hezbullah et al., 2016).

Ingestion of overwhelming metals tainted sustenance brings about diminishing immunological protection, inabilities related with hunger, neurological scatters, intrauterine development impediment, hindered psychosocial conduct and high commonness of upper gastrointestinal growth (Salama, 2018). Over the past few years, numerous studies have been conducted to identify the contamination levels of lead, nickel, cadmium, chromium, arsenic and mercury etc. in cocoa raw materials and chocolate products. For instance, the Rapid Alert System for Food and Feed (RASFF) has been continuously notifying about the presence of excess amounts of heavy metals in cocoa-based food products from the past few years (RASFF Portal, 2016). Highest amounts of $\mathrm{Pb}, \mathrm{Cd}$ and $\mathrm{Ni}$ were found in chocolate products, which contained high cocoa i.e. $(\geq 70 \%$ ) (Sager, 2012). A report on $\mathrm{Cd}$ dietary exposure was published in 2012 by the European Food Safety Authority (EFSA), which highlighted that the high levels of $\mathrm{Cd}$ were found in chocolate products among all other food products contributing the highest dietary exposures in all age groups.

$\mathrm{Pb}, \mathrm{Ni}, \mathrm{Cd}$ and $\mathrm{Cr}$ are naturally occurring elements that are present in a human body at trace levels. Ingestion of $\mathrm{Pb}$ in a human body can cause saturnism poisoning, which is associated with the symptoms such as weight loss, anemia, lead colic, neurological impairments, kidney and liver damage and many others (Montes-Santiago, 2013).

Similarly, Ni also possesses deleterious health effects by exhibiting its biological activity at even ingesting low levels. Its elevated concentrations can accumulate in 
kidney and lungs causing permanent damage to these organs. Several types of cancers like lungs, kidneys, stomach, throat and nose cancers are also associated with consuming Ni. However, its short-term exposure causes some kind of allergic reactions in human body (Sankhla and Kumar, 2019). Moreover, ingestion of $\mathrm{Cd}$ induces reactions in the form of free ions that disturbs many metabolic reactions occurring in body. For example, transformation in proteins, thiamine, and calcium and potassium metabolism can occurs through $\mathrm{Ni}$ poisoning. Oral ingestion of $\mathrm{Cr}$ is also highly toxic to human health; however, its toxicity mainly depends on the oxidation state of Cr (Amjad et al., 2020). Cr (III) is usually considered as non-toxic, whereas $\mathrm{Cr}$ (VI) is highly toxic that can absorbed by gastrointestinal tract in case of oral intake. $\mathrm{Cr}(\mathrm{VI})$ is carcinogenic contaminant that causes DNA damage and gene mutations (Kruszewski et al., 2018; Amjad et al., 2020).

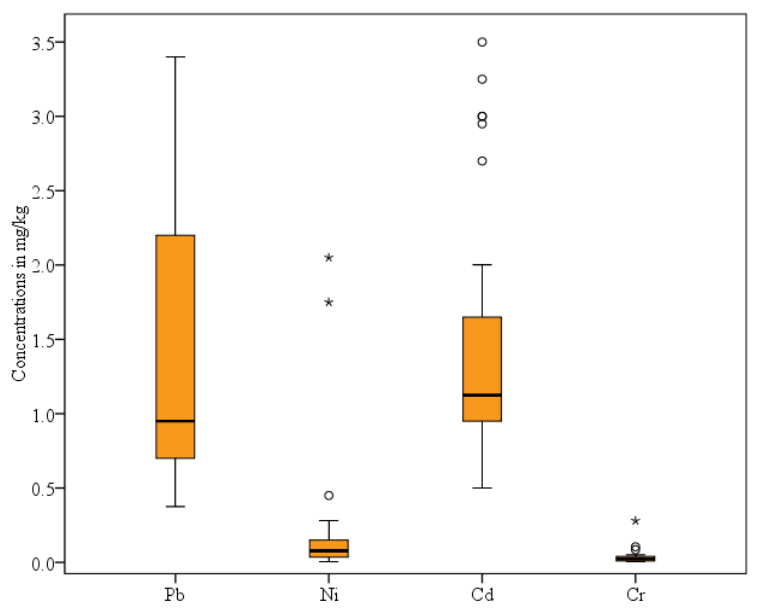

Figure 2. Box plots of $\mathrm{Pb}, \mathrm{Ni}, \mathrm{Cd}$ and $\mathrm{Cr}$ concentrations showing overall statistics of the observed data

Several researches conducted on heavy metal contamination in raw cocoa or chocolate products found that many sources are responsible to increase their levels. The heavy metals could be introduced in the chocolate products at any stage of manufacturing processes like fermentation, drying or using metal instruments for crushing, mixing and packing. Commonly, pressing of cocoa into powder and fat contaminates the products with heavy metals (Salama, 2018). Hydrogenated plant oil is also one of the major sources of heavy metals in chocolates. Commonly, cocoa beans do not contain larger content of heavy metals naturally, so it usually comes from other sources. Lead, nickel, cadmium and chromium may come through drain solids, sugars, coca solids, coca spread, hydrogenated vegetable oil, vegetable fats, permitted emulsifier, buffering specialist, stabilizers, sugar, shading, Sugar, glucose, desiccated coconut powder, sweet whey powder, skimmed drain, powder, emulsifier soya lecithin, simulated sustenance enhance (Zeinali et al., 2019).

According to the available literature, many factors also influence the levels of heavy metals in chocolate products such as geological location of cocoa plant, use of pesticide and/or fertilizers, overall pollution level of the cultivated area and storage conditions. Along with the presence of heavy metal content, their bioavailability plays a significant role to pose its impacts. Apart from the heavy element content in cocoa and chocolate products, their bioavailability is also very important. The health risks of cocoa products are directly associated with low age and weight of consumer. Therefore, studies have shown that children are at the higher risk of heavy metal poisoning in case exceeding the daily intake limit. For example, studies indicate that if $\mathrm{Pb}$ ingested by the digestive track in an adult will cause around $10 \%$ adsorption, while it will be several folds higher in children as 30 to $75 \%$ (Järup, 2003; Abt and Robin, 2020).

Results of 30 samples of local chocolates showed that the highest concentration of lead was $3.75 \mathrm{mg} / \mathrm{kg}$ and lowest concentration of lead was $0.375 \mathrm{mg} / \mathrm{kg}$. Several workers have worked on concentrations of lead in candies and chocolates, the result indicated high levels of lead in sugar based candies (Dahiya et al., 2009). Furthermore, in a total of 30 chocolate samples, the highest concentration of nickel was $2.05 \mathrm{mg} / \mathrm{kg}$ and lowest concentration of nickel was $0.005 \mathrm{mg} / \mathrm{kg}$. Several workers (Dahiya et al., 2005; Jalbani et al., 2009) have worked on nickel concentrations in candies and chocolates, the result indicated the value of nickel is normal in sugar based candies.

Among all 30 samples of local chocolates, it was found that the highest concentration of cadmium was $3.25 \mathrm{mg} / \mathrm{kg}$ and lowest concentration of cadmium was $.5 \mathrm{mg} / \mathrm{kg}$. Several workers (Dahiya et al., 2005; Jalbani et al., 2009) have worked on concentration of cadmium in candies and chocolates, the result indicated the value of cadmium is high in candies and chocolates.

Among all 30 samples of local chocolates it was found that the highest concentration of chromium was $0.085 \mathrm{mg}$ / $\mathrm{kg}$ and lowest concentration of chromium was $.005 \mathrm{mg} / \mathrm{kg}$. Several workers (Dahiya et al., 2005; Jalbani et al., 2009) has worked on concentration of chromium in candies and chocolates, the result indicated the value of chromium is high in candies and chocolates.

The dietary admission of nickel does not prompt any wellbeing hazard in the overall public. Albeit irksome to some sharpened people, an average oral admission of nickel has not been set up. Like lead, nickel levels in cocoa chocolates were higher than in the milk-based chocolates and sugar or organic product seasoned confections. The dietary commitment of nickel has been accounted for to go from 200 to $900 \mathrm{mg} /$ day (Schroeder, 1965; Myron et al., 1978; Clemente et al., 1980; Nielson and Flyvholm, 1984; Smart and Sherlock, 1987; Larsen et al., 2002). In Indian nourishments, the nickel substance announced by Krishnamurti and Pushpa (1991) is a lot higher (240-3900 $\mathrm{mg}$ /day). The commitment of nickel content from chocolates will not influence the everyday admission as the day by day admissions from chocolate will be 55.26 $\mathrm{mg} /$ day (cocoa-based), $34.78 \mathrm{mg} /$ day (milk-based) and $8.68 \mathrm{mg} /$ day (sugar-based confections), if $20 \mathrm{~g}$ of chocolate is taken by any person.

Cadmium gathers in the body primarily in the kidneys and the liver, having a half-existence of quite a few years. The dangerous impact happens in the kidneys and may prompt proteinuria. PTWI has been set up at $7 \mathrm{mg} / \mathrm{kg}$ of body weight (FAO/WHO, 1989). For a tyke with a body weight of $15 \mathrm{~kg}$, the PTWI would be $105 \mathrm{mg} /$ week. In the event that somebody ingests $20 \mathrm{~g}$ cocoa-based chocolate every day, it will contribute roughly $33.5 \%$ of PTWI. Since 
chocolates are not the real nourishment things the cadmium admission from different sources like sustenance and water is probably going to surpass PTWI for youngsters. If there should be an occurrence of milk-based and sugar confections the admission of cadmium is considerably less than cocoa-based chocolates.

There are no well-characterized limits for these components in chocolates in the greater part of the nations aside from a couple. The most extreme degree of lead in chocolate characterized by FAO/WHO (2001) is $1 \mathrm{mg} / \mathrm{kg}$. In Poland, the Polish national standard for lead in chocolates is $0.30 \mathrm{mg} / \mathrm{kg}$ and for cadmium $0.05 \mathrm{mg} / \mathrm{kg}$ (FAO/WHO, 2001). As per these cut off points set somewhere around the Poland administrative office, 42 chocolates out of 69 contained fixations higher than as far as possible.

For instance of cadmium, 19 chocolates contained more than the recommended focus level. The sources of tainting for these metals are for the most part the crude materials utilized, fabricating procedures and filtering of these metals from the vessels where they are put away, particularly the ones still being used that have lead bearing seals. Handling of chocolates is done in steel compartments from which nickel sullying is conceivable notwithstanding the tainting from the impetus utilized in arrangement of the HVO.

\section{Conclusions}

This study demonstrates that locally available chocolates contain heavy metals with high concentrations. 4 toxic heavy metals were selected for the analysis in the current research, which are lead, nickel, cadmium and chromium. A total of 30 representative chocolates samples were purchased from the local markets of Lahore. All the analyses were effectively carried out by using Atomic Absorption Spectrophotometer system at PSCIR Laboratories. The overall results indicated that the levels of all those toxic metals are alarmingly higher in the chocolate samples posing a serious risk to the health of consumers. It is noted that lead is the most abundant toxic heavy metal between other 3 metals, as 90\% samples contain lead concentrations exceeding the PFR permissible levels, which is highly toxic particularly for children because of their low body weight and higher rate of absorption in the digestive track. Lead causes neurological and cognitive impairments in children, lowering the body growth and many others.

As naturally cocoa beans do not contain higher concentrations of heavy metals, their presence is mainly attributed to the manufacturing processes of cocoa products. There are several contamination sources during manufacturing such as storage, raw materials handling, carriage, transportation and the premises for storage of these raw materials etc. Manufacturing equipment are also the main reasons of heavy metals contamination in the chocolates during manufacturing. And unhygienic conditions of the premises and unhygienic conditions of the workers who take part in the formulations of chocolates. Therefore, it is conclude that observed elevated concentrations of heavy metals are due to the non compliance of cGMP (current Good Manufacturing Practices) at manufacturing units working at local level.

\section{References}

Akhtar, S. 2015. Food safety challenges-a Pakistan's perspective. Critical reviews in food science and nutrition, 55(2): 219-226.

Abnet C. C. 2007. Carcinogenic food contaminants. Cancer investigation, 25(3): 189-196.

Alagić N, Huremović J. 2015. Determination of metal contents in various chocolate samples. Glas. Hem. Technol. Bosne Herceg, 45: 39-42.

Abt E, Robin L. P. 2020. Perspective on cadmium and lead in cocoa and chocolate. Journal of agricultural and food chemistry, 68(46): 13008-13015.

Amjad M, Hussain S, Javed K, Khan A. R, Shahjahan, M. 2020. The Sources, Toxicity, Determination of Heavy Metals and Their Removal Techniques from Drinking Water. World, 5(2): 34-40.

Clemens S Ma J F. 2016. Toxic heavy metal and metalloid accumulation in crop plants and foods. Annual review of plant biology, 67: 489-512.

Dahiya S, Karpe R, Hegde A, Sharma R. 2005. Lead, cadmium and nickel in chocolates and candies from suburban areas of Mumbai, India. Journal of Food Composition and Analysis, 18(6): 517-522.

Dapul H, Laraque D. 2014. Lead poisoning in children. Advances in pediatrics, 61(1): 313-333.

Devi P, Bajala V, Garg V. Mor S, Ravindra K. 2016. Heavy metal content in various types of candies and their daily dietary intake by children. Environmental monitoring and assessment, 188(2): 86 .

Dohnalova L, Bucek P, Vobornik P, Dohnal V. 2017. Determination of nickel in hydrogenated fats and selected chocolate bars in Czech Republic. Food chemistry, 217, 456-460.

Godbersen L, Duijnisveld W, Utermann J, Gäbler H, E, Kuhnt G, Böttcher, J. 2012. Application of groundwater thresholds for trace elements on percolation water: a case study on percolation water from Northern German Lowlands. Journal of environmental quality, 41(4): 1253-1262.

Goldstee R, Goldsteen K, Dwelle T. 2014. Introduction to Public Health: Promises and Practice: Springer Publishing Company.

Fong I. 2017. Animals and Mechanisms of Disease Transmission. Emerging Zoonoses, 15-38.

Gizaw Z. 2019. Public health risks related to food safety issues in the food market: a systematic literature review. Environmental health and preventive medicine, 24(1): 1-21.

Hezbullah M, Sultana S, Chakraborty S, Patwary M. 2016. Heavy metal contamination of food in a developing country like Bangladesh: An emerging threat to food safety. Journal of Toxicology and Environmental Health Sciences, 8(1): 1-5.

Iwegbue C. M. 2011. Concentrations of selected metals in candies and chocolates consumed in southern Nigeria. Food additives and Contaminants, 4(1): 22-27.

Iwegbue C. M, Nwozo S. O, Overah C. L, Bassey F. I, Nwajei, G. E. 2013. Concentrations of selected metals in some readyto-eat-foods consumed in southern Nigeria: estimation of Dietary intakes and target hazard quotients. Turkish Journal of Agriculture-Food Science and Technology, 1(1): 1-7.

Jalbani N, Kazi T. G, Afridi H. I, Arain, M. B. 2009. Determination of toxic metals in different brand of chocolates and candies, marketed in Pakistan. Pakistan Journal of Analytical \& Environmental Chemistry, $10(1 \& 2): 5$.

Järup L. 2003. Hazards of heavy metal contamination. British medical bulletin, 68(1): 167-182.

Kirk M. D, Pires S. M, Black R. E, Caipo M, Crump J. A, Devleesschauwer B, Döpfer D, Fazil A, Fischer-Walker C. L, Hald T. 2015. World Health Organization estimates of the global and regional disease burden of 22 foodborne bacterial, protozoal, and viral diseases, 2010: a data synthesis. PLoS medicine, 12(12): e1001921. 
Kruszewski B, Obiedziński M. W, Kowalska, J. 2018. Nickel, cadmium and lead levels in raw cocoa and processed chocolate mass materials from three different manufacturers. Journal of Food Composition and Analysis, 66: 127-135

Kanmani S, Gandhimathi R. 2013. Assessment of heavy metal contamination in soil due to leachate migration from an open dumping site. Applied Water Science, 3(1): 193-205.

Lari S. Z, Khan N. A, Gandhi K N, Meshram T. S, Thacker N. P. 2014. Comparison of pesticide residues in surface water and ground water of agriculture intensive areas. Journal of Environmental Health Science and Engineering, 12(1): 1-7.

Leiter J, Harding S. 2004. Trinidad, Brazil, and Ghana: three melting moments in the history of cocoa. Journal of rural Studies, 20(1): 113-130.

Montes-Santiago J. 2013. The lead-poisoned genius: saturnism in famous artists across five centuries. Progress in brain research, 203: 223-240.

Malakootian M, Nekoie Moghdam M, Jannati A, Pourshaaban Mazandarany M. 2012. Determination of Lead Levels in Candy, Chocolate and Chewing Gum Existent in Kerman in 2009 and Compare it's by FDA Standard: A Short Reaport. Journal of Rafsanjan University of Medical Sciences, 11(2): 179-184.

Mohammed FS, Akgul H, Sevindik M, Khaled BMT. 2018. Phenolic content and biological activities of Rhus coriaria var. zebaria. Fresenius Environmental Bulletin, 27(8): 56945702 .

Mohammed FS, Pehlivan M, Sevindik M. 2019. Antioxidant, antibacterial and antifungal activities of different extracts of Silybum marianum collected from Duhok (Iraq). International Journal of Secondary Metabolite, 6(4): 317-322.

Mohammed FS, Günal S, Şabik AE, Akgül H, Sevindik M. 2020. Antioxidant and Antimicrobial activity of Scorzonera papposa collected from Iraq and Turkey. Kahramanmaraş Sütçü İmam Üniversitesi Tarım ve Doğa Dergisi, 23(5): 1114-1118.

Nordberg G. F, Nogawa K, Nordberg, M. 2015. Cadmium Handbook on the Toxicology of Metals. UK: Academic Press. ISBN 978-0-12-398292-6

Odeyemi O. A, Sani N. A, Obadina A. O, Saba C. K. S, Bamidele F. A, Abughoush M, Asghar A, Dongmo F. F. D, Macer D, Aberoumand A. 2019. Food safety knowledge, attitudes and practices among consumers in developing countries: An international survey. Food research international, 116: 13861390.

Ochu J, Uzairu A, Kagbu, J, Gimba C, Okunola O. 2012. Evaluation of Some Heavy Metals in Imported Chocolate and Candies Sold in Nigeria. Journal of Food Research, 1(3): 169.
Olmedo P, Pla A, Hernández A, Barbier F, Ayouni L, Gil, F. 2013. Determination of toxic elements (mercury, cadmium, lead, tin and arsenic) in fish and shellfish samples. Risk assessment for the consumers. Environment international, 59: 63-72.

Ramtahal G, Yen I. C, Bekele I, Bekele F, Wilson L, Sukha B, Maharaj K. 2015. Cost-effective method of analysis for the determination of cadmium, copper, nickel and zinc in cocoa beans and chocolates. Journal of Food Research, 4(1):193.

Rehman S, Husnain, S. M. 2012. Assessment of trace metal contents in chocolate samples by Atomic Absorption Spectrometry. J. Trace Elem. Anal, 1(1): 1-11.

Rivas L, Mellor G. E, Gobius K, Fegan, N. 2015. Detection and Typing Strategies for Pathogenic Escherichia coli. Australia: Springer New York Heidelberg Dordrecht London. ISBN 978-1-4939-2345-8

Sankhla M. S, Kumar, R. 2019. Contaminant of Heavy Metals in Groundwater \& its Toxic Effects on Human Health \& Environment, 18 (5): 01-05.

Saha R, Nandi R, Saha B. 2011. Sources and toxicity of hexavalent chromium. Journal of Coordination Chemistry, 64(10): 1782-1806.

Salama A. K. 2018. Health risk assessment of heavy metals content in cocoa and chocolate products sold in Saudi Arabia, 38(4): 318-327

Sevindik M, Akgul H, Pehlivan M, Selamoglu Z. 2017. Determination of therapeutic potential of Mentha longifolia ssp. longifolia. Fresen Environ Bull, 26(7): 4757-4763.

Sevindik M. 2019. The novel biological tests on various extracts of Cerioporus varius. Fresenius Environmental Bulletin, 28(5): 3713-3717.

Temiz H, Soylu A. 2012. Heavy metal concentrations in raw milk collected from different regions of Samsun, Turkey. International journal of dairy technology, 65(4): 516-522.

Turan G. Y. M, Akccedil F. 2011. The importance of Turkish hazelnut trace and heavy metal contents for human nutrition. Journal of Soil Science and Environmental Management, 2(1): 25-33.

Verna R. 2013. The history and science of chocolate. The Malaysian journal of pathology, 35(2): 111.

Villa J. E, Peixoto R. R, Cadore, S. 2014. Cadmium and lead in chocolates commercialized in Brazil. Journal of agricultural and food chemistry, 62(34): 8759-8763.

Yanus R. L, Sela H, Borojovich E. J, Zakon Y, Saphier M, Nikolski A, Karpas Z. 2014. Trace elements in cocoa solids and chocolate: an ICPMS study. Talanta, 119(15):1-4.

Zeinali T, Salmani F. Naseri K. 2019. Dietary intake of cadmium, chromium, copper, nickel, and lead through the consumption of meat, liver, and kidney and assessment of human health risk in Birjand, Southeast of Iran. Biological trace element research, 191(2): 338-347. 